Estudios Románicos, Volumen 28, 2019, pp. 115-126

ISSN: 0210-491

eISSN: 1989-614X

DOI: https://doi.org/10.6018/ER/377621

\title{
MARCEL SCHWOB, EPISTOLARIO
}

(Marcel Schwob, collection of letters)

\author{
Francisco Ríos Romero* \\ Universidad de Cádiz
}

\begin{abstract}
This article shows and analyzes the interesting correspondence that Marcel Schwob kept up with his family, friends and many writers with he stablished an epistolary relationship on account of his writing profession. Marcel Schwob had a relationship with the most prominent writers and artists in his time, among them, Verlaine, Mallarmé, Valéry, Claudel, Gourmont or Jarry; above all, it was an unrepeatable period for the literature and the art. It will make a special about all reference to R. - L. Stevenson because his works had an important influence on Schwob who considered Stevenson as a master. The letters sent to his family and to Marguerite Moreno, with he married and who stayed with him to his last day, present a simple man, a close person with daily worries and suffering from a serious disease.
\end{abstract}

Keywords: Schwob; Works; Letters; Travels; Friends; Love.

Resumen: El presente artículo muestra y analiza la interesante correspondencia que mantuvo Marcel Schwob con su familia, amistades y otros escritores con los que estableció una relación epistolar debido a su oficio de escritor. Marcel Schwob tuvo una relación con los escritores y artistas más destacados de su tiempo en un periodo irrepetible para la literatura y el arte en general, entre ellos encontramos a Verlaine, Mallarmé, Valéry, Claudel, Gourmont, Jarry. Mención aparte requiere R.-L. Stevenson, al que Schwob consideraba su maestro y que tanto influyo en su obra. Las cartas a su familia y a Marguerite Moreno, con la que contrajo matrimonio y le acompañó en sus últimos días, revelan un hombre sencillo, una persona cercana, con sus preocupaciones cotidianas y angustiosamante afectado por su enfermedad.

Palabras Claves: Schwob; Obras; Correspondencia; Viajes; Amigos; Amor.

\footnotetext{
*Dirección para correspondencia: Francisco Ríos Romero (friosrom@gmail.com).
} 
En este artículo presentamos un breve análisis de la producción epistolar de Marcel Schwob, escritor, ensayista y traductor francés, considerado hoy en día por algunos escritores y críticos como uno de los grandes precursores de la literatura francesa actual. Autor de relatos, cuentos, estudios lingüísticos y artículos periodísticos, gozó de cierto prestigio entre los autores de su generación que admiraban su singularidad estética. Una muerte prematura le impidió desarrollar una obra más extensa que es poco conocida en la actualidad salvo excepciones como Vies Imaginaires o Le livre de Monelle. En este estudio abordamos el corpus epistolar de este autor dividido en dos grupos, en un primer grupo analizaremos su correspondencia familiar y sentimental; incluimos en este grupo un apartado dedicado al intercambio epistolar con escritores, editores y amigos; a algunos de estos destinatarios se les puede atribuir estas tres cualidades. Estas últimas cartas tienen un contenido literario e intelectual, propias del oficio de escritor, en ellas encontramos opiniones, referencias, críticas, recomendaciones y agradecimientos entre escritores. Existe otro grupo que no son cartas al uso, es decir, textos que no son el resultado de la comunicación escrita entre personas sino que se trata de literatura, escritos periodísticos "artículos" y otros textos de crítica literaria publicados en diferentes periódicos que fueron titulados por Schwob "Lettres", Lettres Parisiennes y Lettres à Valmont, aunque no todos tengan forma de cartas y que por lo tanto no incluiremos en este estudio.

Marcel Schwob nació en una familia de intelectuales, su padre George Schwob fue condiscípulo de G. Flaubert, amigo de T. Banville, T. Gautier y J. Verne con quien escribió alguna pieza de teatro, dueño y editor del periódico Le Phare de la Loire, donde Schwob publicó su primera reseña con once años. Mathilde, su madre era descendientes de rabinos y médicos. Su tío Léon Cahun, escritor y antropólogo, jefe de la biblioteca Mazarine donde Schwob vivió durante un tiempo mientras se iniciaba de la mano de su tío en el camino de la erudición y el misterio de los archivos. En una carta de 1988 dirigida al editor Hetzel proponiéndole la traducción de La flecha negra de Stevenson, sin duda uno de los escritores que más le influyeron, Schwob presenta algunos de los escritores anteriores como referencias de su preparación: «Dans notre courte entrevue, je n'ai pu vous donner aucune référence. M Michel Bréal, professeur au collège de France, mon oncle M. Léon Cahun chez qui j'habite et M. Jules Verne pourront vous parler de moi» (Schwob 1985:112)

La correspondencia que mantuvo Schwob con sus familiares y amigos no se encuentra publicada en su totalidad, una gran parte de ella permanece inédita depositada en diferentes archivos y bibliotecas aunque afortunadamente desde hace unos años está siendo objeto de estudio por parte de investigadores. Las bibliotecas e instituciones donde están depositadas la mayor parte de sus cartas y otros documentos son: la Bibliothèque Nationale de France, la Bibliothèque Littéraire Jacques Doucet, la Bibliothèque de l'Institut de France, la Bibliothèque de l'Arsenal, la Bibliothèque Municipale de Nantes y la Brigham Young University (Utah, U.S.A.).

La primera y más completa edición de parte de la correspondencia de Schwob hasta el momento es la realizada por el profesor John Alden Green con el título, Correspondance Inédite, (Droz, 1985) fruto de sus trabajos de investigación en los que reunió 
más de trecientas cartas escritas por Schwob entre 1877 y 1904. J. A. Green realizó una selección de estas cartas con precisas anotaciones e interesantes comentarios sobre personajes y otros aspectos relevantes para entender el contexto de las mismas. Además de las cartas, esta obra incluye una selección de textos inéditos como artículos y cuentos publicados en diferentes periódicos como L'Événement o L'Écho de Paris, así como otros documentos como la partida de nacimiento de Schwob, el certificado de su matrimonio con Marguerite Moreno o fotos del escritor en diferentes momentos de su vida. Incluyó el autor una extensa y detallada bibliografía, la más completa hasta la fecha. Aunque esta obra sea la primera edición de cartas escritas por Schwob, se debe de tener en cuenta que la primera recopilación de determinada correspondencia que Schwob mantuvo con otros escritores y amigos fue incluida por Pierre Champion, su discípulo, en la biografía que consagró a su maestro, Marcel Schwob et son temps (Grasset Editeur, 1927). En este apéndice titulado "Le livre de l'amitié", encontramos además de anécdotas, opiniones y retazos de la vida de Schwob, extractos de cartas et "billets" que intercambiaron otros escritores como Jules Renard, Paul Claudel, Colette y Francis Jammes con Schwob, escritores que colaboraron en aquellos días en Mercure de France. En esta revista Schwob escribió un artículo titulado La perversitél donde hace referencia a L'Écornifleur, obra escrita por Renard. Schwob escribe estas bellas palabras a propósito del personaje:

L'Écornifleur est un jeune homme dont le cerveau est peuplé de littérature. Rien pour lui ne se présente comme un objet normal. Il voit le XVIIIè siècle à travers Goncourt, les ouvriers à travers Zola, la société à travers Daudet, les paysans à travers Balzac et Maupassant, la mer à travers Michelet et Richepin. Il a beau regarder la mer. S'il aime, il se rappelle les amours littéraires. S'il viole, il s'étonne de ne pas violer comme en littérature. Sa tête est pleine de fantômes. (Schwob 1896: 221).

En agradecimiento, Renard le remitió una carta fechada el 26 de febrero de 1892 con las siguientes palabras: «Mon cher ami, que je vous serre la main pour votre bel article $\mathrm{du}$ Mercure. Comme c'est haut. Cela donne même un peu de vertige. Tout un univers en sept pages. Je vous assure que l'Écornifleur sent sa tête tourner. Il est habitué déjà aux petits articles terre à terre. Et, d'un coup, vous le lancez en plein ciel...» (p. 249).

En otra ocasión es Schwob quien le remite unas palabras para consolar a Renard que en otra carta anterior le confesaba su descontento por su falta de inspiración y por el resultado final de su obra Poil de Carotte. Lo interesante en la correspondencia entre estos dos escritores es el contenido literario de las mismas así como las opiniones y comentarios que encontramos sobre sus propias obras y sobre las de otros escritores. Renard admiraba la obra de Schwob, en una carta del 29 de junio de 1894 escribe sobre Le Livre de Monelle: «Ce que je vous dis plus facilement, c'est que le Livre de Monelle m'a donné une joie très rare, spéciale, et qu'il m'a pris ces jours-ci les meilleures de

$1 \quad$ Este artículo fue publicado en Mercure de France, 1892, IV y recogido junto con otros artículos y prefacios de otras obras de Schwob en Spicilège, 1896, Mercure de France, pp. 211-221. 
mes heures». La misma admiración expresa por La Croisade des enfants, «ce petit livre joli tout plein, et ingénieux, et attendrissant...J'aime beaucoup lire Schwob» y por Vies imaginaires, «Avant de fermer cette lettre je viens de lire la préface des Vies. C'est une page admirable que tous les artistes devraient apprendre par cœur. Pour moi elle m'enchante et me raffermit", escribe en una carta del 23 de junio de 1896.

La amistad entre estos dos escritores que empezó en 1891 cuando Schwob fue a pedirle a Renard un cuento para L'Écho de Paris, incluso Schwob le dedicó el cuento "La machine à parler" incluido en Le Roi au masque d'or (1892), fue diluyéndose en el tiempo, al parecer desde el momento en que Schwob empezó su relación con Marguerite Moreno con la que contrajo matrimonio en 1900, hasta el punto que la opinión de Renard sobre la obra de Schwob ha cambiado de forma radical como podemos comprobar en un comentario en su Journal fechado el 7 de junio: "Le talent de Schwob, c'est une mixture de vins, ce n'est pas un vin. Je me moque de cette intelligence. Tous ses contes, il les a empruntés."’2.

Otra gran amistad inalterable en el tiempo aunque perjudicada por la distancia es la que mantuvo Schwob con Paul Claudel que fueron compañeros de clase en el Lycée Louis-le-Grand, "Mais Schwob admirait Claudel; et Claudel admirait Schwob", escribe Pierre Champion. Cuando Schwob termina la lectura de Tête d'or de Claudel (1891) no duda en comunicarle por carta su admiración: "Je ne crois avoir lu rien de plus beau ces dernières années", a esta carta le sigue algunos días después otra en el mismo tono de emoción: "Mon cher Claudel, Plus je relis ton livre, plus j'en suis enthousiasmé. [...] je sentais quelque chose d'extraordinairement fort, et tout à fait extérieur à moi. Voici que maintenant il me saisit tout à fait. [...]".

Del mismo nivel son los elogios que Claudel escribe sobre Le Roi au masque d'or; en esta obra Schwob dedica a su amigo el cuento apocalíptico tiulado "L'incendie terrestre": "[...] Voilà mon cher Schwob, bien des raisonnements, et tout cela pour ne pas te dire le plaisir que me fait ton livre. [...] Je suis extrêmement fier de t'avoir pour ami..." (Champion 1927: 262). Dos años más tarde Claudel, que se encuentra destinado en Boston como diplomático, responde con sus comentarios poéticos quasi metafísicos al envió de Mimes (1894):

[...] Comme je te l'ai déjà dit, la caractéristique de ton style me semble être le nom et le nominatif [...] De là une impression de réalité dans l'inexistant qui ne figure pas mal le fait de l'odeur [...] J'aime mieux louer l'œuvre qui existe comme d'elle-même et comme une image qu'on touche [...] Adieu mon bon Schwob. Je te remercie de l'amitié que tu montres pour ma sœur; tu es un véritable ami pour nous. Pourquoi ne m'écris-tu pas, misérable homme? Espèce de coquillart! (Champion 1927: 264-265)

Schwob remitirá a Claudel otras obras como su traducción de Hamlet que Claudel consideró admirable o un ejemplar de La Croisade des Enfants cuyo envío agradeció en

2 Las citas de las cartas de Jules Renard han sido extraidas del libro de Pierre Champion sobre Schwob. Extractos del Journal pueden ser consultados en: Journal de Jules Renard de 1899-1904. textes.libres;free.fr/français/jules-renard_journal-de-jules-renard-de-1899-1904.htm. 
una carta con fecha, 21 de abril de 1900: «Et ce goût amer et subtil, cette secrète sensibilité, cette pitié de ce qui n'est plus, qui m'a fait aimer Mimes, me font aimer plus encore La Croisade des Enfants» (Champion 1927: 270)

Estos son algunos ejemplos de la variada correspondencia literaria que recoge Pierre Champion en la biografía sobre Schwob, posteriormente en Correspondance Inédite, J. A. Green publica un gran volumen de correspondencia remitida por Schwob a otros escritores entre los que se encuentran André Gide, Anatole France, Octave Mirbeau, Jean Lorrain, Paul Valery, Paul Léautaud o Stéphane Mallarmé.

Schwob mantuvo con André Gide una relación cordial a partir de 1891 (Gide recomendó a Schwob la lectura de Tête d'or) que se enfriaría en 1897 cuando Gide publica Les Nourritures terrestres, obra que en determinados pasajes presenta bastante similitud con Le livre de Monelle, y comienza entonces cierta polémica en el mundillo literario por el posible plagio de Gide $^{3}$. En la que parece ser la primera carta remitida por Schwob a Gide, le hace referencia a la lectura de "votre remarquable livre Les Cahiers d'André Walter [...] - leur triste histoire m'étreignait tandis que je vous lisais. Aussi je n'aime pas votre livre - mais il est beau, tout de même. Je serais heureux de faire votre connaissance" (Schwob 1985: 115). A finales de ese mismo año Gide visita a Schwob en su apartamento en rue de l'Université y quedó impresionado por su aspecto, como escribe en su Journal. A pesar de la polémica, Schwob siempre trato a Gide de "cher ami" como podemos leer en algunas de sus últimas cartas; en una de ellas, fechada en 1903 opinando sobre L'Inmoraliste, "un beau livre que j'aurais voulu encore plus beau. Mais c'est une œuvre qui marque fort et qu'on imitera", en otra escrita también en el mismo año, le transmite su opinión sobre Prétextes, libro que ha leído "avec grand plaisir" y donde agradece a Gide la recomendación de la lectura de In Memoriam dedicado a Oscar Wilde, donde Gide presenta a Wilde "tel que je l'ai connu et tous ceux qui l'ont aimé et admiré vous auront de la reconnaissance."4

Sin dudar del interés de toda la correspondencia seleccionada, nada más que el nivel literario de los receptores como P. Valery, S. Mallarmé, A. France u Octave Mirbeau a los que trata de "maître et ami" aconseja una lectura pausada y detallada. En una de las cartas de 1903 dirigida a Mirbeau, que por entonces era miembro de l'Académie Goncourt, le recomienda la obra de Paul Léautaud, Le Petit Ami, para recibir el premio de 1904. Leautaud consideraba a Schwob un maestro y amigo, luego, como en otras ocasiones, el alumno comenzó a criticar al maestro, infravalorando su obra: "Cher maître et ami, voulez-vous me permettre de vous recommander de toute mon énergie pour le Prix Goncourt Le Petit Ami de Paul Léautaud, dont je crois déjà vous avoir parlé. Je ne crois pas que la récompense puisse être mieux placée ni plus utilement..."

3 Gide nunca entendió las acusaciones de plagio y admitió reconocer las semejanzas con el libro de Schwob que estima ser superior al suyo. En la entrada correspondiente al 20 de septiembre de 1931 de su Journal, Gide escribe sobre este asunto a modo de confesión, declarando que Les Nourritures terrestres es el más espontaneo y sincero de todos sus libros.

$4 \quad$ Gide escribe este libro cuando, durante su estancia en Argel, se entera de la muerte de Wilde. Schwob al igual que Gide y otros escritores del círculo de Mercure de France mantuvieron una estrecha relación de amistad con el escritor inglés cuando vivió en Paris. Schwob incluso dedicó a Wilde "Le Pays bleu", uno de los cuentos incluidos en Le Roi au masque d’or; esta relación llegó a su fin cuando comenzó el proceso judicial contra Wilde. 
Léautaud criticaba en la obra de Schwob una excesiva erudición que rayaba en la pedantería y que estropeaba algunas de sus mejores páginas hasta el punto de escribir con fecha 14 de julio de 1903 en su Journal Littéraire ${ }^{5}$ la siguiente crítica acerca del estilo de Schwob:

Mon opinion depuis longtemps sur la littérature de Schwob. Au fond, très au fond, je n'y trouve aucun intérêt. C'est de la fabrication, de la marqueterie et je sens comment c'est fait et avec quoi. De vastes lectures, dans tous les genres, - des phrases et des idées notées sur des fiches, - puis arrangement, combinaison de ces phrases et de ces idées classées par catégories, en un tout quelconque [...]. C'est truqué au possible. S'il y a un grand écrivain aujourd'hui... c'est Barrès et Gourmont...les autres Schwob, France, Régnier, etc...sont des truqueurs, rien de plus.

La lectura de esta correspondencia entre Schwob y todos estos escritores contemporáneos de tanta relevancia para la historia de la literatura es de gran interés para poder realizar un retrato de la escena literaria "fin-de-siècle" lo que quizá relega a un segundo plano las cartas dirigidas a su familia, con un contenido menos relevante desde una perspectiva literaria pero fundamentales para poder seguir el desarrollo personal del autor y conocer sus inquietudes e ideas sobre numerosos temas. Las primeras cartas de esta correspondencia las escribe Schwob a la edad de diez años y a partir de ese momento vamos a conocer al escritor como bachiller, militar voluntario, escritor debutante, universitario, colaborador en prensa o conferenciante. Nos advierte Green que durante un periodo de cuatro años, desde septiembre de 1892 (el padre de Schwob fallece en agosto) hasta junio de 1896 no existen cartas dirigidas a su familia periodo que coincide con la publicación de sus primeras obras de relevancia, Cour Double (1891), Le Roi au masque d'or (1892), Mimes (1893), Le Livre de Monelle (1894). En estos años Schwob comienza a vivir su vida adulta y conoce a Louise, inspiradora de Monelle, cuya muerte prematura sumió al escritor en una gran desolación. El segundo bloque de cartas familiares, desde 1896 hasta 1904, tiene a la madre del escritor como principal destinatario y un tema frecuente como es la enfermedad del escritor que falleció en 1905.

Como parte de la correspondencia familiar pueden ser incluidas las cartas dirigidas por Schwob a su mujer Marguerite Moreno. Estas cartas están claramente divididas y editadas en tres grupos, el primero de ellos es una selección de cartas que recoge Champion en el capítulo X de la biografía de Schwob, "Du prisme de l'amour au prisme de la maladie" donde se describe, a principios del año 1895, el comienzo de esta relación amorosa. Schwob conoció a Marguerite, actriz del Théâtre Français et de la Comédie Française que años después también interpretaría con éxito algunos papeles en el cine, en la redacción de L'Écho de Paris donde visitaba a Catulle Mendès, redactor jefe de este periódico. Champion describe de esta manera el nacimiento de este amor sublime: "Marcel Schwob, qui ne pouvait connaître que des sentiments extrêmes, l'aima d'une manière absolue, la suivant partout; il pleurait en l'écoutant dire des vers, approuvait

$5 \quad$ Journal Littéraire, Vol. I (1893-1928), 1986, Mercure de France. 
tout d'elle, délirant devant sa gracile beauté, ses longs cheveux dénoués" (Champion 1927: 108). En estas cartas Schwob se muestra poseído en cuerpo y alma por un amor total que le anula todos los sentidos y le limita su personalidad:

Il y a longtemps que je ne t'ai écrit, mon âme chérie, et cela me fait de la peine. Je suis triste dès que je suis loin de toi...Mon âme, je suis affreusement jaloux...Je t’aime si tendrement, si dévotement. Je parle comme toi maintenant, et mon écriture va ressembler à la tienne sans que je le fasse exprès. Quand tu t'en vas, je tombe dans une espèce de torpeur. - Mais toi, tu vis autrement de ta vie, ma chérie. Et cela me fait beaucoup souffrir. [...]

Je m'ennuie, je m'ennuie, Marguerite, sitôt que je ne suis plus avec toi. Que faire - c'est affreux - je sens que je ne dormirai pas cette nuit. J'ai ta fièvre. Je te parle pendant que je ne dors pas: je te dis que je t'aime, que je t'aime. Oui, je t'aime éperdument. J'ai parlé de l'amour avant toi, mais je ne savais pas ce que c'est. Je sens que je pourrais être assassin, voleur et incendiaire. Et j'ai peur, j'ai peur, parce que je ne me sens pas digne de toi. [...] Mon adorée, je ne sais ce que je t'écris, j'ai la fièvre d'amour. Pardonne-moi si ce que je te dis est mal. Je te jure que je ne sais pas ce que je fais. Je voudrais être plus intelligent pour mieux te comprendre, pour savoir tout. [...]

Je tremble encore en t'écrivant. Tu te rends compte de ce que tu as fait? Tu m'as tué; je n'existe plus qu'en toi. [...] Je ne peux pas te dire que je t'aime. - Ce n'est pas assez fort; je meurs de toi, et tu me fais mourir de toi. Écrase-moi sur tes pieds. MARCEL (Champion 1927: 109-114)

Esta angustiosa relación epistolar culmina el doce de septiembre de 1900 en Londres donde Marguerite Moreno y Marcel Schwob contrajeron matrimonio; a partir de estos primeros años del siglo XX la enfermedad afecta de manera progresiva la salud del escritor.

En busca de alivio para su enfermedad y quizá intuyendo que ese podría ser el último gran viaje de su vida, decide realizar un viaje a Samoa (Pacífico Sur) para visitar la tumba del que considera su gran maestro R.-L. Stevenson. A este periodo, comprendido entre octubre de 1901 y marzo de 1902, en el que tiene lugar el viaje, corresponde el segundo grupo de cartas dirigidas a Marguerite; cartas que están recogidas y publicadas con el título, Le voyage à Samoa. Lettres à Marguerite Moreno (octubre 1901-mars 1902) de las que Champion hace una selección en el capítulo XVI de la biografía de Schwob, "Le voyage a Samoa (Octobre 1901- Mars 1902)". Champion escribe en la introducción que las cartas originales fueron entregadas por Marguerite a Maurice el hermano de Marcel, pero que estas se perdieron y las publicadas son unas copias abreviadas de las originales hechas por Lucie, hija de Maurice y futura escritora y artista conocida como Claude Cahun. Champion confirma la veracidad de estas cartas porque él llegó a tener en sus manos las cartas auténticas remitidas por Schwob, "Mais je les ai tenus entre mes mains. Je vois encore le papier fin, la petite écriture menue et un peu pâteuse qui se déroule sous le roseau. Ces notes étaient prises par lui-même" (Schwob 
1930:8) ${ }^{6}$. Además de estas cartas, Schwob toma notas y apuntes para escribir un libro sobre el viaje que nunca verá la luz, en sus notas a modo de un cuaderno de abordo recoge sus impresiones sobre los paisajes, los viajeros, la tripulación y otros problemas cotidianos como la enfermedad de su criado chino Ting o la falta de dinero para hacer frente a sus gastos, sin dejar de escribir literatura y sobre literatura. Este viaje se convertirá para Schwob en un viaje al infierno pues está a punto de morir a causa de una fiebre de $40^{\circ}$ por neumonía que le afecta poco antes de su destino en Apia; las altas temperaturas, su debilidad y los mosquitos ponen su salud al límite. Después de una dura singladura y ya instalado en Samoa escribe el diez de enero: "Un peu fatigué, ma Marg dorée; je viens d'écrire à Maurice. Je suis cloué: je n'ai pas plus un sou et il faut que je revienne tout de suite. Je ne supporte pas le climat. [...] Je t'adore, je t'adore je ne pense plus qu'à toi, ma chérie, ma femme - pourvu que vous me fassiez vite revenir !" (p. 271). El veinticinco de enero, escribe quizá la carta más angustiosa al comentar detalles de su enfermedad:

Ma Marg adorée,

J'ai été terrassé par la fièvre vers le 8 janvier et j'ai cru mourir. Je n'avais plus ma tête. J'allais de mon lit à la douche pour faire baisser la température qui pendant douze jours a oscillé entre $39^{\circ} 8$ et $40^{\circ} 5$. Je me traînais sous l'eau froide parce que je voulais te revoir. [...] Et pendant ce temps j'avais une pneumonie droite sans savoir autre chose sinon que je soufrais là et ne pouvais respirer. [...] En fin la température a cédé un peu. Mais j'étais et suis encore un squelette et ne puis-me tenir debout. Oh! ma chérie, j'ai si peur de mourir sans revoir ta figure chérie! J'ai eu du courage. J'ai été sous l'eau froide en grinçant des dents et tout ce que j'endurais de la chaleur, des moustiques et des mouches, cela ne se peut dire. Mais je voulais te revoir et revenir chez nous. (pp. 271-272)

El veintiocho de enero de 1902, en el viaje de vuelta a Francia, Schwob se encuentra algo mejor pero se asfixia y necesita ayuda para todo, incluso le cuesta trabajo escribir pero la escritura le distrae de su enfermedad y le alivia porque le acerca a su amada Marguerite:

[...] Ma chérie, j'ai été triste aujourd'hui; mais j'ai prié à Dieu de tout mon cœur qu'il me permette de revoir et d'embrasser ta chère figure. Vois-tu, je n'ai pas de force, et je ne peux t'écrire parce qu'il me semble que je te parle et que tu es là. Jamais la distance ne m'autant terrifié qu'en ce moment. Si Dieu permet que je te revienne, que je revoie maman et Maurice et notre maman et tous les nôtres, j'aurai vécu plus que la vie de douleur d'un homme. Après tout ce que j'ai traversé de souffrances, voici l'angoisse la plus affreuse qui m'a été réservée. Je prie Dieu que ce soit la dernière et la plus grande épreuve. Mes épaules plient sous les faix de

6 Marcel Schwob, 1927-1930, Euvres Complètes, vol. 10, éd. Pierre Champion, Bernouard, Paris. Los textos de las cartas de Voyage à Samoa, corresponden a esta edición. 
la douleur, j'ai eu plus que ma part. Mais je t'ai, mon adorée, il faut que je pense à cela. C'est peut-être en compensation que je souffre tout cela. Chère souffrance, alors ! Je reprendrai courage pour revoir ma Mag chérie. Je le veux de toute ma volonté et de tout mon cœur (c'est grâce à mon cœur qui m'a soutenu que j'ai résisté à la terrible fièvre.) (pp. 277-278)

Schwob continuó enfermo y con medicamentos todo el tiempo que duró la travesía hasta Marsella el 20 de marzo de 1902, donde llegó con el convencimiento de nunca más viajar: "Jamais plus je ne m'en irai!..."

El otro grupo de cartas abarca los últimos meses de la vida de Schwob desde octubre de 1904; en este periodo el estado de salud del escritor empeora progresivamente mientras que su amada Marguerite se encuentra de gira por los Paises Bajos. Estas cartas están incluidas al final de Correspondance Inédite y entre ellas encontramos las últimas cartas, notas y "billets" dirigidas por Schwob a su mujer Marguerite Moreno bajo el epígrafe "Les dernières lettres à sa femme". En esos momentos la actividad literaria de Schwob es mínima y por la enfermedad se siente incluso fatigado para realizar cualquier trabajo intelectual, aunque está preparando un curso en la Sorbonne sobre Villon y su época al que asistirán ilustres alumnos como Paul Fort, Max Jacob o Picasso entre otros.

En esta época la relación con Marguerite es algo distante y en estas cartas escribe sobre el día a día, su enfermedad y otros problemas cotidianos como la falta de ingresos, pero en ellas encontramos también referencias literarias y comentarios sobre el mundo del teatro así como felicitaciones por los éxitos y críticas favorables que recibe Marguerite durante la gira. El 19 de febrero de 1905 Schwob escribe la última carta a su mujer:

Ma chérie, merci de ta bonne lettre et je suis bien content que tu aies la lécithine. [...] Bravo pour ton grand succès devant le grand duc que je lis dans le Figaro [...] Tu ne m'as pas envoyé l'article, ni à Hérold. J'ai fait déranger Hérold deux fois pour cela. Tu n'es pas gentille. Où est-il cet article? Je suis très grippé. A demain. Kisses; Ton Marcel. Je pense que tu m'auras envoyé de l'argent. Je suis à bout. Tu ne devrais pas me laisser ainsi, ma chérie. Je serais plus tranquille, si j'avais un peu d'avance... (Green 1985: 208-209)

Para finalizar esta aproximación al legado epistolar de Marcel Schwob, es obligada referencia, la breve pero significativa relación que mantuvo el escritor con el autor que él mismo consideró su gran maestro y hasta cierto punto responsable de haber emprendido el viaje por el camino de la escritura, Robert Louis Stevenson. El primer contacto de Schwob con el escritor escocés se produce en su juventud cuando en el

7 Marguerite Moreno estaba interpretando la obra de Octave Mirbeau, Les affaires sont les affaires con la compañía Hertz Coquelin.

El artículo al que hace se hace referencia era un encargo que Schwob le había hecho a Marguerite, que también escribía, sobre el pintor flamenco Jan van Scorel, uno de los pintores preferidos de Schwob. 
verano de 1884 lee Treasure Island y queda cautivado por este libro, como escribe en su ensayo sobre Stevenson publicado en Spicilège: “Alors je connus que j'avais subi le pouvoir d'un nouveau créateur de littérature et que mon esprit serait hanté désormais par des images de couleur inconnue et des sons point encore entendus" (Schwob 1896: 98-99) $)^{8}$

La relación entre los dos escritores fue únicamente epistolar a pesar de algunas propuestas de encuentros que nunca llegaron a producirse, Stevenson había estado dos veces en Francia, viajes que realizó en los años 1878 y 1879, de lo que dió testimonio con dos relatos de estos viajes, pero después nunca tuvo otra ocasión pues sus pasos le llevaron a Estados Unidos y luego al Pacífico donde sabemos falleció. En el año 1888, Schwob tenía 22 años cuando escribió por primera vez a Stevenson; en su carta se presenta como un estudiante de "licence", admirador de su obra, ofreciéndole un manuscrito para que escribiera una novela que luego él traduciría y donde habla de sus afinidades literarias:

Nous avons le même goût pour les vieux gentilshommes poudrés, polis, fermes, intelligents, à l'esprit clair du dix-huitième siècle. Vous aimez ce siècle et je l'aime aussi. Nous avons les mêmes principes de composition, je veux dire la simplicité et au lieu de la description coordonnée qu'emploient les réalistes, une description progressive avec quelques traits marquants qui représentent beaucoup plus un tableau pour l'œil de l'esprit qu'une analyse précise et détaillée. Enfin, ce n'est pas la moindre chose, les personnages que vous créez sont tellement semblables à la vie, ils se meuvent si aisément et parlent si naturellement qu'il me semble avoir vécu quelques heures avec John Silver et Alan Breck et que je ne puis faire autrement que de vous aimer pour cela (Champion 1927: 162)

En febrero de 1889, Stevenson le responde desde Honolulu:

Cher Monsieur,

Je vous remercie - au milieu de toute cette agitation que vous pouvez imaginer, avec sept mois de correspondance accumulée sur ma table - pour vos deux lettres amicales et intelligentes. Merci de m'écrire encore. Je serais chez moi en mai ou juin, et je viendrai probablement à Paris pendant l'été. [...] Votre petit conte est délicieux. Ah oui, vous avez raison, j'aime le dix-huitième siècle; tout comme vous, qui n'avez pas entendu sa voix en vain. - Celui Que l'on Poursuit. Robert-Louis Stevenson (Schwob-Stevenson 1992: 47)

Schwob continua leyendo a Stevenson, The Black Arrow, Kidnapped y en una carta le propone a Stevenson la traducción de La Flecha Negra. Schwob ya había obtenido

8 Champion escribe en la biografía de Schwob que lo que este admiraba en Stevenson era la visión particular que tenía de la vida: "Mais ce qui transportait d'admiration M. Schwob, ce qui lui rendait Stevenson si cher, c'était son art d'évocation; il admirait le poète qu'il était vraiment, avec son point de vue particulier sur la vie, beaucoup plus que les aventures que Stevenson a pu conter." (p.159) 
la aprobación de los editores ingleses para esta traducción, pero Stevenson le responde en una larga y cordial carta el 19 de agosto de 1890 que no es una buena obra: "Vous désirez traduire La Flèche Noire! Cher Monsieur, la présente vous y autorise; mais je vous avertis que je n'aime pas cet ouvrage." En esta misma carta le habla de la edición de su obra, Mers $d u$ Sud, para la que su ahijado irá a Paris en busca de ilustraciones ya que él no podrá viajar:

Hélas! Je n'aurai pas le plaisir de vous voir encore de quelque temps, si même je puis vous voir jamais. Il faut vous contenter de m'accepter comme une voix errante et sous forme de lettres occasionnelles venant d'îles mystérieuses; adressez les vôtres, si vous êtes assez bon pour m'écrire, à Apia, Samoa.

En una carta enviada desde Sydney en 1891, Stevenson le agradece el artículo que sobre él ha publicado Schwob en octubre de 1890 en L'Événement et Le Phare de la Loire y le aconseja traducir como mejor opción David Balfour o Le Maître de Ballantrae, sin descartar Le Prince Othon: «Il faut que vous me traduisiez vite; vous aurez bientôt mieux à faire qu'à transvaser l'ouvrage des autres».

La última carta de Stevenson a Schwob data del 7 de julio de 1894, en ella le agradece el envío y le comenta la agradable lectura de Mimes: "C'est un livre gracieux, essentiellement gracieux, avec sa hantise d'agréable mélancolie, son aimable saveur d'antiquité. En même temps par ses mérites, il se montre la promesse de quelque autre chose à venir, plutôt qu'une chose finale en soi." (Schwob-Stevenson 1992: 53)

En diciembre de 1894 R.-L. Stevenson fallece con 44 años en Valima (Samoa); lamentaba Schwob en la necrológica publicada en Le Phare de la Loire una muerte tan temprana, cómo iba a imaginar que la muerte le esperaba algo más tarde con 37 años.

\section{BIBLIOGRAFÍA}

CHAMPION, Pierre (1927): Marcel Schwob et son temps. Paris: Bernard Grasset, Editeur.

GIDE, André (1986): Journal Littéraire, Vol. I (1893-1928) Paris: Mercure de France.

LÉAUTAUD, Paul (1986): Journal Littéraire, Vol. I (1893-1928) Paris: Mercure de France.

SCHWOB, Marcel (1896): Spicilège. Paris: Mercure de France. (1927-1930): CEuvres Complètes. Vol. 10. Éd. Pierre Champion. Paris: François Bernouard Éditions.

(1985): Correspondance Inédite, précedée de quelques textes inédits. Édition John Alden Green. Genève: Librairie Droz.

STEVENSON, Robert-Louis (1992): Will du moulin, suivi de M. Schwob/R.-L. Stevenson: Correspondances. Édition établie par François Escaig. Paris: Édition Allia. 


\section{PERFIL ACADÉMICO Y PROFESIONAL}

Licenciado en Filosofía y Letras. Filología Francesa (Universidad de Granada)

Master Estudios Hispánicos (Universidad de Cádiz)

Profesor Asociado de Francés. Universidad de Cádiz (1997-2000)

Doctorando (Universidad de Cádiz)

Investiga sobre la biografía imaginaria en Marcel Schwob y otros autores.

Fecha de recepción: 18/05/2019

Fecha de aceptación: 12/07/2019 